Huerta Jiménez, Mayra.

Profesor-Investigador. Facultad de Artes. Cuerpo Académico Imagen y Creación, UABC.

Vicari Zanatta, Claudia.

Profesor-investigadora. Instituto de Artes. Grupo de investigación Ciudadanía y Arte, UFGRS.

\title{
La mediación artística en comunidades de mujeres con riesgo vulnerabilidad social a partir del audiovisual.
}

\section{Artistic mediation through audiovisual work in communities of women with risk of social vulnerability.}

\author{
TIPO DE TRABAJO: \\ Comunicación virtual. \\ PALABRAS CLAVE: \\ Audiovisual, mujeres, vulnerabilidad social, mediación artística.
}

KEYWORDS:

Audiovisual, women, social vulnerability, artistic mediation.

RESUMEN.

El audiovisual cobra cada vez más relevancia en la actualidad, puesto que permite demostrar argumentos significativos que involucran emociones que no son sencillas de enunciar, permitiendo narrativas que simulan y atestan realidades complejas.

En este sentido, las mujeres que pasan por procesos violentos en ciertas ocasiones se puede tomar una distancia para evidenciar aspectos más controlados ahora en un ambiente más familiar y de cooperación; si están en este tipo de ambientes, o por el lado contrario enfrentándose todos los días a su situación cotidiana en continua vulnerabilidad.

El propósito de este ensayo es mostrar el proyecto "Porto Alegre-Tijuana: mujeres mirando su vida cotidiana y más allá" en una red de colaboración entre grupos de investigación: Ciudadanía y Arte (UFGRS, Brasil) ${ }^{1}$ e Imagen y Creación ${ }^{2}$ (UABC, México). Los dos grupos vienen trabajando en colaboración desde el año 2014, relacionando prácticas artísticas con el objetivo principal de compartir y desarrollar metodologías de investigación en artes ${ }^{3}$.

Se planteó una aproximación a la cotidianidad de esas mujeres de estos dos grupos focales a partir de la utilización del video como herramienta de comunicación, ya que este promueve un acercamiento más íntimo desde la mirada de las

\footnotetext{
${ }^{1}$ Ciudadanía e Arte es un grupo de investigación vinculado a la Universidad Federal do RS e actúa como práctica artística crítica en el espacio urbano. Del grupo participan alumnos y profesores desde distintas áreas del conocimiento. Coordinación general Prof. Cláudia Vicari Zanatta. Informaciones respecto al Ciudadanía e Arte disponibles en el sitio en internet: cidadaniaearte.wix.com/ufrgs

${ }^{2}$ Imagen y Creación, conformado por profesores de la Facultad de Artes de la Universidad Autónoma de Baja California (UABC), México. El grupo desarrolla proyectos de creación a partir de una reflexión de diversas disciplinas como lo son: la escultura, la fotografía, el vídeo, la pintura, la grafica e intervención en el espacio con arte urbano.

${ }^{3}$ Detalles respecto a actividades colaborativas entre los dos grupos pueden ser encontrados en: http://paralelos28e30.wixsite.com/arte
} 
propias participantes y a la vez narra situaciones que las constituyen a partir de sus especificidades. Aspectos que el video formula desde el lenguaje audiovisual.

\section{ABSTRACT.}

Audiovisual gains more relevance nowadays, because it enables the demonstration of significant arguments that involve emotions that aren't easy to enunciate, allowing narratives that simulate and attest to complex realities.

In this sense, women that go through a violent process, on certain occasions, can take a distance to evidence more controlled aspects in a now more familiar and cooperative environment; if they are in that type of environment, or on the contrary, facing their everyday situation on a daily basis in continued vulnerability.

The purpose of this essay is to show the proyect "Porto Alegre-Tijuana: Women looking at their everyday life and beyond" in a network of collaboration between research groups: Citizenship and art (UFGRS, Brasil) and Image and Creation (UABC, Mexico).

In adition to this, the procedure consisted in both cases on approaching this group of women that share risk situations (domestic violence, low income, low education and with difficulty entering the workforce). In the case of Porto Alegre, work was done with women who live in a low income neighborhood, with favela (shanties) characteristics. In the case of Tijuana, work was done with women from the rehab center Casa Corazón. During several months we dialogued through several video workshops that resulted in various audiovisual works made by the participants which speak of their lives and also of the cities they live in.

An approximation to the daily lives of these two focal groups of women was proposed through the utilization of video as a communication tool, because it promotes a more intimate approach from the point of view of the participants themselves and at the same time narrates situations that constitute them based on their own specificities. Aspects that video formulates from an audiovisual language.

\section{CONTENIDO.}

Introducción.

En el año 2015, Ciudadanía y Arte e Imagen y Creación han sido contemplados en el programa Iberamericano IberCultura Viva, cuyo enfoque es apoyar redes de cooperación e intercambio que fortalezcan culturas de base comunitaria en países ibero-americanos. ${ }^{4}$ Con la aportación financiera proveniente del programa, fue desarrollado el proyecto Porto Alegre- Tijuana: mujeres mirando su vida cotidiana y más allá. El proyecto tuvo como objetivo fomentar la aparición de narrativas como una manera de mostrar la historia y la vida cotidiana de algunas de las mujeres que viven en los suburbios de las dos ciudades, a partir de su propia mirada. La actividad del vídeo fue la herramienta elegida para generar el intercambio de experiencias, valoración de los sujetos y de sus capacidades, permitiendo a los participantes establecer una red fruto de las particularidades locales y culturales de esos dos contextos muy distintos. Se considera que el video promueve un acercamiento más íntimo desde la mirada de las propias participantes y a la vez narra situaciones que las constituyen a partir de sus especificidades.

El procedimiento consistió en ambos casos en acercarse dos grupos de mujeres que comparten situaciones de riesgo (violencia doméstica, pocos recursos financieros, algunas con muy baja escolaridad y con dificultad para entrar en el mercado del trabajo). En el caso de Porto Alegre se trabajo con mujeres que habitan en un barrio de baja renta, con características de favela (chabolas). En el caso de Tijuana, se trabajo con mujeres en el Centro de Rehabilitación Casa Corazón. Durante varios meses se dialogó a través de talleres de video que derivaron en diversos audiovisuales realizados por las participantes, los cuales hablan de su vida y también de las ciudades en que viven.

Aproximaciones a la mediación artística.

La mediación es un concepto relativamente nuevo, dentro de la práctica artística. En una diversidad de países; este concepto se ha desarrollado de diversas formas, de acuerdo a sus condiciones y limitantes de uso, tanto geográficas, contextuales y políticas. En este aspecto es significativo recalcar el uso de las políticas y el incidir en contextos que no son propiamente claros para la mediación, sino

\footnotetext{
${ }^{4}$ Concepto y amplias informaciones sobre el programa IberculturaViva disponible en: http://iberculturaviva.org
} 
todo lo contrario está mediación esta relacionada con el ejercicio de las políticas públicas y las prácticas artísticas colaborativas. Como lo menciona Kester, Grant (2009):

"Uno de los rasgos destacados de la práctica artística colaborativa contemporánea es la creciente permeabilidad entre la producción artística y otras prácticas culturales y formas organizativas. El trabajo de grupos activistas, movimientos sociales y ONG ofrece un punto de contigüidad particularmente importante, así como de diferenciación".

Estos proyectos de los que menciona Kester, se pueden revisar en Transductores ${ }^{5}$ proyecto cultural realizado en Centro José Guerrero de Granada. Las propuestas están realizadas desde varios ejes de investigación enfocada a esta práctica colaborativa y pedagogías colectivas de trabajo en red. Involucra a 13 grupos internacionales que articulan la práctica artística, la intervención política y la educación. Al revisar esta literatura, es importante identificar en que contexto se encuentra en relación al proyecto y sus actores que se esta planteando.

La mediación como lo define en su artículo Maria Lind ${ }^{6}$ curadora es una trasferencia:

Vermittlung - "mediation" in German-signifies a transfer from one party to another, the pragmatic transmission of a message. It also stands for attempts at reconciling parties who disagree on something: nations, for instance, or people in conflict. Although there is an abundance, even an overproduction, of traditionally didactic activities within art institutions today, I believe that now is the time to think more and harder about the mediation of contemporary art (Lind, 1998:99).

Se propone la curaduría como comisario de esta mediación o transferencia, a partir de eventos educativos, sociales, políticos y económicos con el aspecto curatorial para pensarlo en el proyecto "Porto Alegre-Tijuana: mujeres mirando su vida cotidiana y más allá". Al relacionarlo de esta forma se integra la investigación en el proceso curatorial en la actualidad. De la misma manera el proyecto, esta sustentado en una parte curatorial como eje de la reflexión educativa que se desarrolla para el trabajo en colaboración desde una postura de construcción del conocimiento fomentando la participación colectiva definiendo al Arte, como "agente de cambio" en los aspectos que logra en cada participación de las involucradas.

El dispositivo videográfico para la realización de los talleres.

Por otra parte, "[...] lo videográfico sería un dispositivo de la imagen en movimiento, un tipo de relación de trabajo que promueve una dimensión crítica y autorreferencial basada en la manipulación fácil y directa" (Rodríguez, M.L.2011:15); de acuerdo a la investigación de Lorena Rodríguez Mattalia el vídeo como dispositivo abarca diversos planteamientos para construir una narrativa aportando su versatilidad y aproximación inmediata.

Tal como, el proyecto propuso este acercamiento a la imagen en movimiento con cualquier tipo de cámara a la mano, desde un móvil, hasta una cámara semiprofesional que permita la manipulación para el fin deseado conformado por los talleres que se impartieron como a continuación se describen.

En Porto Alegre, los talleres han sido realizados con mujeres residentes en la Vila Renascença I (local de baja renta situado en zona central de la ciudad). En el local residen principalmente personas afro descendientes en condiciones de vulnerabilidad social (enfrentan situación de vivir con pocos recursos financieros, de violencia doméstica o de prejuicios por su condición social). Todas las mujeres participantes del proyecto (un grupo de 6 mujeres adolescentes) tenían baja escolaridad y hacían parte de familias que reciben auxilio gubernamental mensual por no tener renta mínima que permita la supervivencia.

Desde el año 2015 el grupo Ciudadanía y Arte vienen trabajando en la Vila Renascença $\mathrm{I}^{7}$ y ha sido a partir de ese contacto que se invitó a las moradoras a participar en el proyecto de audiovisual. Han sido realizados durante cuatro meses talleres de vídeos mediante encuentros informales, en los cuales fue dado énfasis a conversaciones respecto a la vida cotidiana de las mujeres en la ciudad de Porto Alegre. Los talleres proporcionan elementos básicos para manipular cámaras de video (primeramente cámaras de celulares, después una cámara compacta y al final una cámara profesional). Al final del proyecto han sido producidos 5 audiovisuales por las participantes. ${ }^{8}$

\footnotetext{
${ }^{5}$ Centro José Guerrero. (2009). Transductores.Pedagogías colectivas y politicas espaciales. Granada, España: Gráfica Alhambra.

${ }^{6}$ Maria Lind is an art critic and curator based in Stockholm. She is currently the artistic director of IASPIS, The International Artists Studio Program in Stockholm. Previously, she held the position of curator at Munich Kunstverein and Moderna Museet. In 1998, she co-curated Manifesta 2. - See more at: http://moussemagazine.it/ten-fundamental-

${ }^{7}$ El grupo desarrolla en la comunidad el proyecto Território llhota. Informaciones disponibles en: http://territorioilhota.wixsite.com/ilhota

${ }^{8}$ Los videos están disponibles en: http://portotijuana.wixsite.com/mulheres/porto-alegre
} 


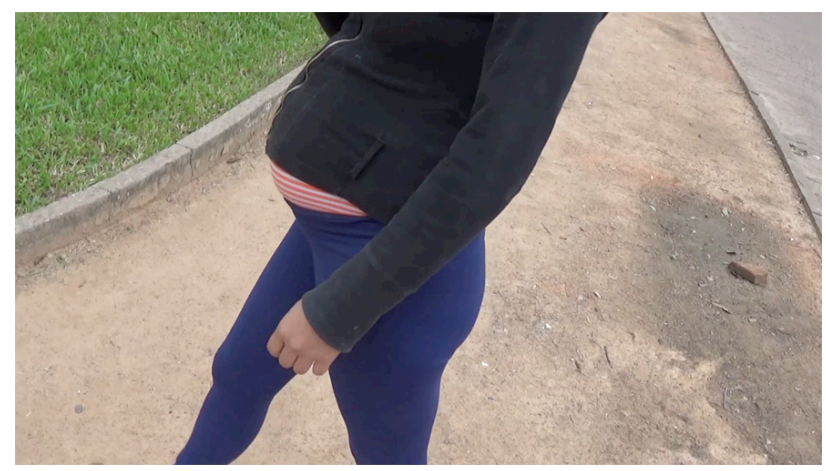

Ilustración 1. Talle de video que impartió Marcia Braga en Porto Alegre, Brasil.

Los vídeos retratan algunos recorridos hechos habitualmente por las participantes en la ciudad de Porto Alegre y algunos recorridos por calles donde ellas jamás habían estado antes. Las mujeres han decidido que caminos recurrir, lo qué y cómo grabar, qué imágenes les gustaría mostrar y lo que decir. Siempre con la cámara en la mano, en movimiento, las grabaciones reflectan lo que cada una ha juzgado importante compartir mediante su mirada, el entendimiento que tienen de su vida en la ciudad, sus preocupaciones con el futuro, deseos, sueños, como también la experiencia de caminar por calles desconocidas. Muchos de esos aspectos son indicados de modo subjetivo, en conversaciones, gestos, posturas corporales, brillo de los ojos, interés o desánimo en relación a temas de su vida cotidiana en la ciudad.

A menudo la cámara ha sido usada como un ojo que recorrió las calles buscando y seleccionando lo que grabar desde distintas sensibilidades. Ella hizo con que la vida cotidiana, banal, automática ganase otro tono por medio de reconocimiento de sus sonidos, olores, luces, otro tiempo (el tiempo del desplazamiento lento, atento al momento presente). La ciudad ha sido como que redescubierta a partir de otros puntos de vista y desde distintas sensibilidades.

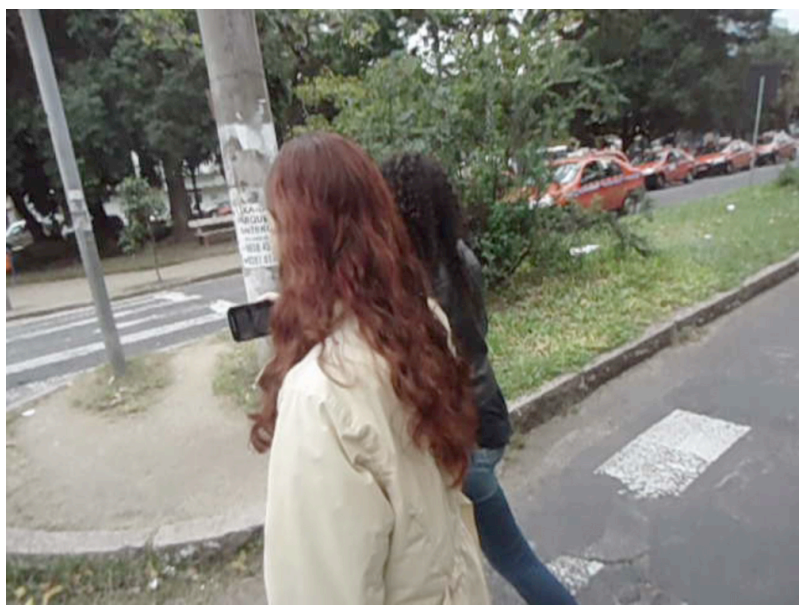

llustración 2. Taller de video impartido por Viviane Gueller en Porto Alegre, Brasil.

En el caso de Tijuana, el taller se impartió en el Centro de Rehabilitación Casa Corazón. Este espacio alberga entre 15 y/ o 20 mujeres que permanecen alrededor de 6 meses, tiempo que dura la estancia. La labor social en este centro, como en la mayoría se enfoca en la reinserción social y el bienestar de las mujeres que deciden cambiar su entorno. 
Para ello, se invitar a la sociedad a participar de forma activa con la donación ya sea monetaria o en especie para que este propósito del programa se cumpla. A partir de establecer el contacto con Alejandra Aduna ${ }^{9}$, artista visual voluntaria que imparte talleres de pintura se estableció el contacto para impartir el taller en este espacio.

El taller planteó como objetivo identificar la comunicación y el video a manera de herramienta para expresar lo que en su actualidad de estas mujeres acontece. En este sentido, lo cotidiano ahora se volvió el concepto a desarrollar. En un inicio se trabajo con 15 mujeres y después se fueron sumando a cada sesión algunas más. Pero al mismo tiempo otras terminaban su tratamiento lo cual implicaba trabajar por sesión para tener un producto tangible que les funcionará tanto a las participantes como al desarrollo del taller.

Las sesiones se orientaron por medio de conceptos básicos de comunicación, lenguaje cinematográfico y videográfico para desarrollar una narrativa de los momentos específicos que vivían en el centro. El eje era el trabajo en equipo, para desarrollar sus historias y representar lo que en ese momento sentían. Temas como la comunicación, el espacio, el retrato y autorretrato y la cotidianidad se construyeron en colectivo.

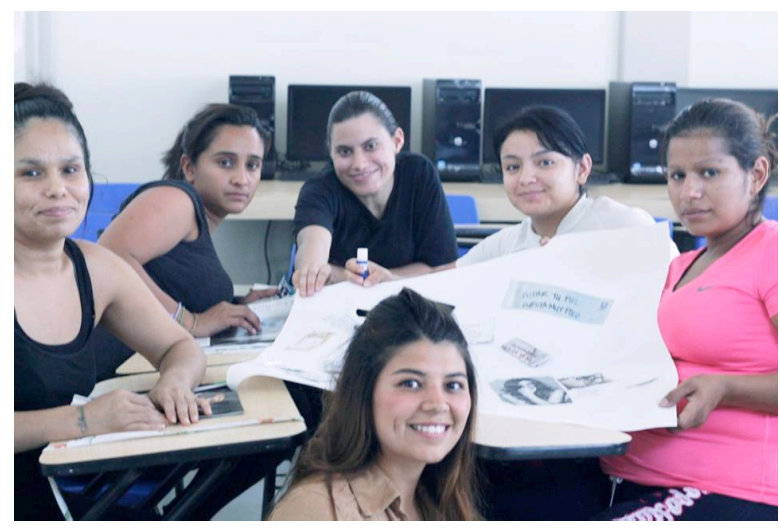

Ilustración 3. Facilitadora María Luisa Chávez, apoyando al equipo para el desarrollo del story board para la grabación en la sesión, Tijuana, B.C.

Durante el taller se tomo como base, un día de actividades que realizan en el espacio. Así como los diversos roles que desempañan por semana. Frente a esto, si les tocaba estar en apoyando en la cocina. La grabación se realizaba en esa área u otro rol estaba en relación a la limpieza, se buscaron las locaciones de grabación con las que se identificaran. Esto con una previa planeación de los espacios donde grabar, así mismo en el taller tenían tareas asignadas; cómo estar frente a la cámara de video o salir como actriz improvisada generando una acción para la cámara. Se organizaron por equipos, y cada equipo tenía asignada a una facilitadora que apoyaba en sus dudas o necesidades técnicas. Cada equipo mostró su narrativa de acuerdo a los momentos que deseaban capturar con la cámara.

Por otra parte, se trabajo con el concepto de autorepresentación a partir del audiovisual retratos. El propósito era reflexionar sobre como se visualizan ellas en general, en ese sentido la forma más directa para autorrepresentarse era salir a cámara sin inhibiciones o la otra postura era tomando una cierta distancia apareciendo poéticamente. Desde la utilización de carteles con texto, objetos, así como elementos que se encontraban alrededor de ellas. Preferían dar prioridad a su cabello, a los lunares o las manos, rasgos físicos que no están propiamente vinculados al rostro. Dando la oportunidad de proyectarse de otra forma acentuando más los aspectos con los que se sentían cómodas. Después de esta experiencia muchas se dieron cuenta que se les facilitaba salir enfrente de la cámara.

\footnotetext{
${ }^{9}$ Alejandra Aduna Reyes-Pérez es maestra en Artes y artista plástica, es fundadora del taller de arte "La llave" desde el año 2000 donde se ha desarrollando como profesora de artes plásticas para niños y adultos desde entonces. Su obra se ha distinguido por un interés particular por la mujer y su entorno, en disciplinas que incluyen pintura, creación sonora y video.
} 


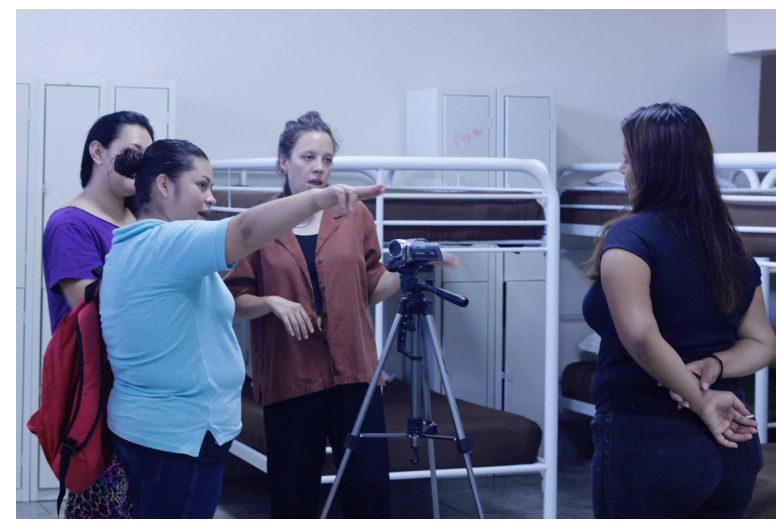

Ilustración 4. Facilitadora María José Crespo, apoyando al equipo para el desarrollo de las escenas de la sesión 3 en Tijuana, B.C.

El tomar la cámara y poder manipularla para ellas, dirigir a sus compañeras y lograr una historia fue algo nuevo pero a la vez importante en su autoestima. Puesto que expresaron lo que sus emociones estaban sintiendo en ese momento. Decirlo para que otro más lo escuche y decida no repetirlo o apoyar en algún sentido.

Por lo tanto, una parte del resultado en el taller logra expresar frente a sus familiares; el contar como se sienten estando en el sitio recuperándose en apoyo con las otras mujeres que comparten su misma condición. Puesto que, al divertirse grabando también se concentraban en narrar mostrar un momento doloroso en transición.

\section{¿Cómo establecer contacto entre las dos realidades? Las muestras de video y el sitio web.}

Cómo mostrar y poner en contacto las dos realidades enfocadas por el proyecto ha sido una de las metas enfrentadas por Porto Alegre-Tijuana. Así, fueron realizadas dos exhibiciones en las dos ciudades con los vídeos producidos ${ }^{10}$. Los trabajos realizados han sido mostrados especialmente a familiares y amigos de las protagonistas. Se pudo conocer un poco de la realidad de los dos contextos, siendo establecida una vía de comunicación entre los dos grupos focales.

En consecuencia, al verse en la grabación tomo momentos de relajación y reflexión sobre su estancia para evidenciar que existen otras maneras de expresar su situación. Y al ver a las mujeres de Porto Alegre (Brasil), saber que no están solas y pueden unirse con otras mujeres.
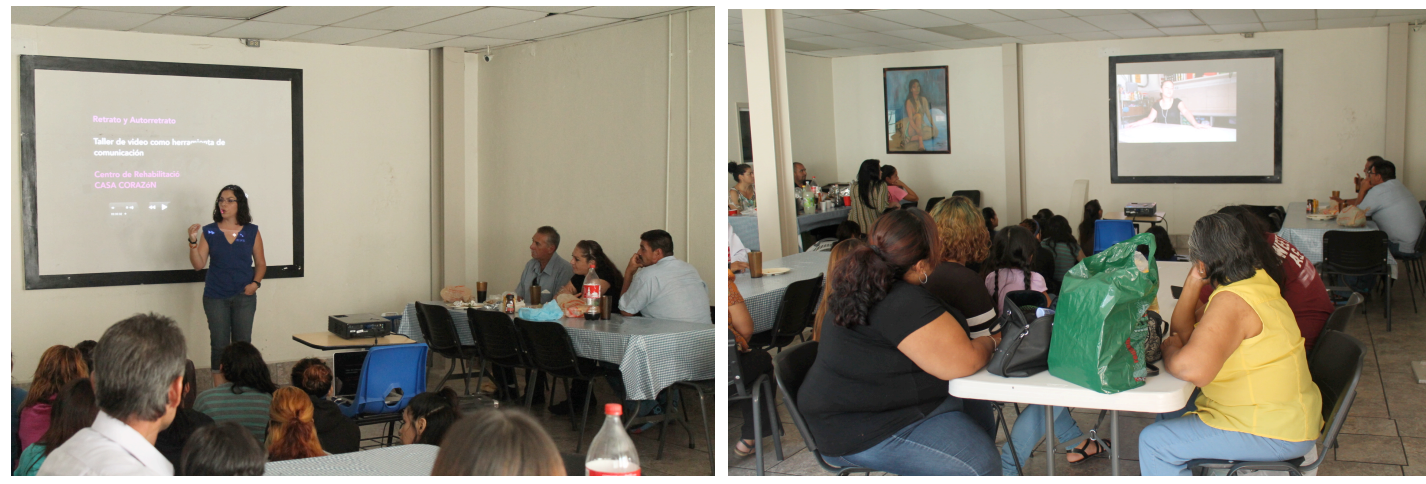

Ilustración 5 y 6. Presentación ante los familiares en el Centro de Rehabilitación Casa Corazón.

\footnotetext{
${ }^{10}$ En Porto Alegre, la exhibición ocurrió en el Centro Municipal Lupicínio Rodrigues y en Tijuana, en la Casa Corazón.
} 
También ha sido producido un sitio web (portugués-español) para divulgar y compartir todos los trabajos generados, ampliando el intercambio de prácticas artísticas urbanas con otros países de lengua portuguesa y española.

El sitio web, también sirvió para divulgación da obra generada y tiene la función de preservar la memoria de los eventos realizados por el proyecto. $^{11}$

\section{Conclusiones:}

El audiovisual ha dado la oportunidad para la emergencia de narrativas como forma de evidenciar la historia y la vida de mujeres que ha menudo no tienen sus actividades cotidianas valoradas. A lo largo de los talleres hemos notado que hubo un compartir de experiencias y que el recurso audiovisual ha potenciado la mirada sobre la propia vida diaria de la ciudad, sea en un ambiente cerrado como es una casa de rehabilitación sea en la libertad de la calle.
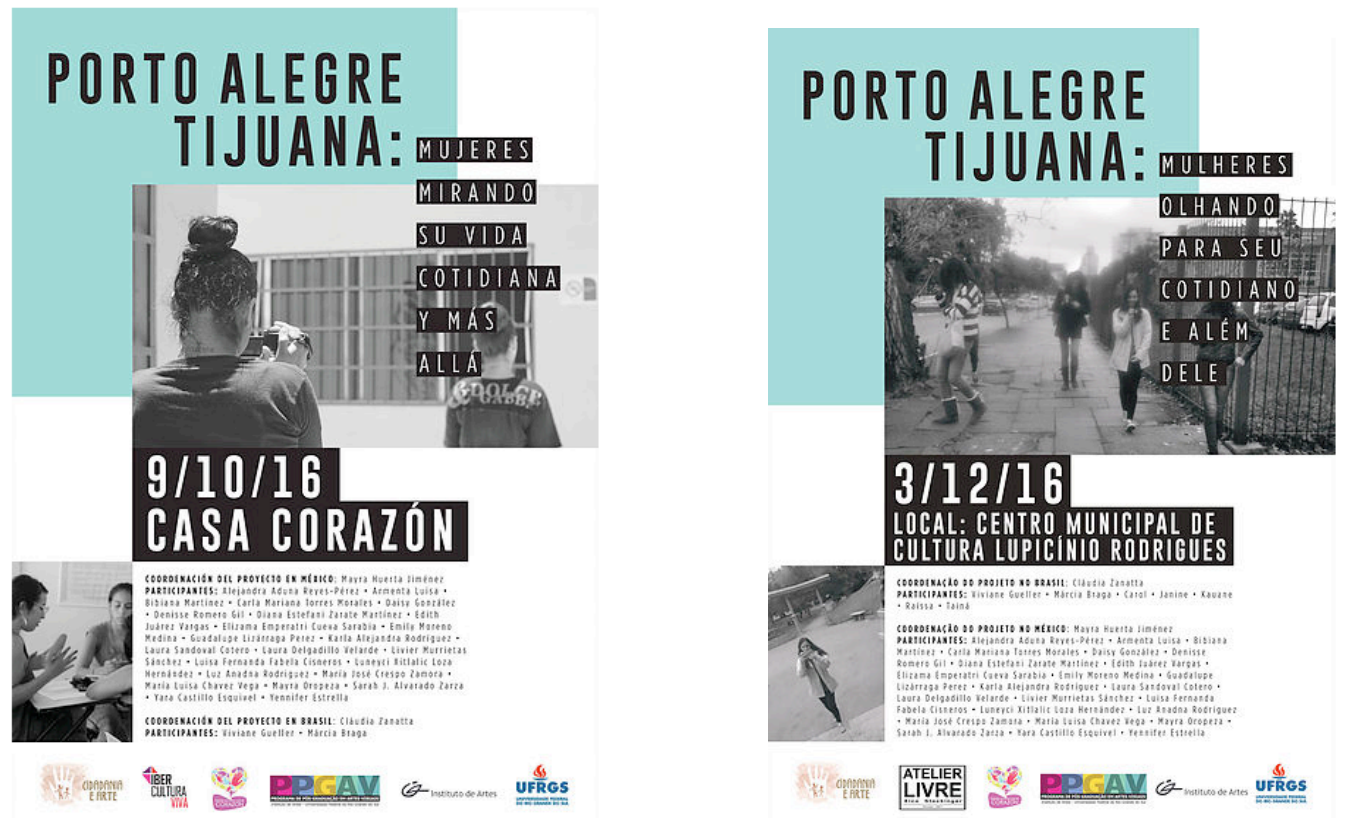

llustración 7. Los carteles de difusión de las muestras de video en ambos contextos (Tijuana-Porto Alegre)

La experiencia de protagonizar la propia historia ha tenido grande valor reflexivo y apuntó a las posibilidades inesperadas que pueden ser encontradas en cotidianidades que a menudo parecen no tener cualquier interés. Cotidianidades que podrían ser llamadas "de paso", como por ejemplo a la de casa de rehabilitación o calles ya tan conocidas. Realizar algo creativo a partir de situaciones precarias, difíciles y dolorosas puede traer ánimo por medio de actividades que más que buscar un fin, buscan un estar juntos, un compartir miradas, sentimientos y modos de ser y estar en el mundo.

El proyecto también contrapuso dos realidades bastante distintas: la de las calles de una ciudad a la de un ambiente cerrado, de privación temporaria de la libertad. Dos locales opuestos, pero con posibilidades de encuentros que muestran cómo es la ciudad del "otro", a partir de su ritmo, de sus experiencias personales. Se descubre así que una ciudad es hecha de muchas otras, tan infinitas y diversas cuanto sus habitantes.

\footnotetext{
${ }^{11}$ El sitio es: http://portotijuana.wixsite.com
} 
Huerta Jiménez, Mayra; Vicari Zanatta, Claudia

La mediación artística en comunidades de mujeres con riesgo vulnerabilidad social a partir del audiovisual

III CONGRESO INTERNACIONAL DE INVESTIGACIÓN EN ARTES VISUALES :: ANIAV 2017 :: GLOCAL [codificar, mediar, transformar, vivir http://dx.doi.org/10.4995/ANIAV.2017.5796

\section{FUENTES REFERENCIALES.}

Centro, J. G. Transductores.Pedagogías colectivas y políticas espaciales. Granada, España: Grafica Alhambra. 2009.

Favre R. Imagem-tempo e imagem-movimento [Internet]. São Paulo: Laboratório do processo formativo [accessed 2 Abr 2016]. http://laboratoriodoprocessoformativo.com/2016/04/imagem-tempo-e-imagem-movimento/\#.Vv64yK9WXNs.facebook

Huerta, J.M., Vincari, Z. C. "Porto Alegre-Tijuana: mujeres mirando su vida cotidiana y más allá"

www.ufrgs.br/secom/ciencia/tag/mulheres-em-vulnerabilidade-social/ (accessed 21 de noviembre de 2016).

Lind, M. Why media art? Ten fundamental questions of curating. Nueva York, E.E.U.U.1998.

Rodríguez M. L. Videografía y arte: indagaciones sobre la imagen en movimiento. Universitat Jaume I. 2011. 